\title{
1 Cross-species analysis of LZTR1 loss-of-function mutants demonstrates 2 dependency to RIT1 orthologs
}

3

5

6

7

9

Antonio Cuevas-Navarro1, Laura Rodriguez-Muñoz ${ }^{2}$, Joaquim Grego-Bessa ${ }^{3}$, Alice Cheng $^{1}$, Katherine A. Rauen ${ }^{4,5}$, Anatoly Urisman ${ }^{6}$, Frank McCormick ${ }^{1}$, Gerardo Jimenez ${ }^{2,7}$, Pau Castel ${ }^{8, *}$

${ }^{1}$ Helen Diller Family Comprehensive Cancer Center, University of California San Francisco, San Francisco, CA (US)

${ }^{2}$ Institute for Molecular Biology of Barcelona, Consejo Superior de Investigaciones Científicas, Barcelona (Spain)

${ }^{3}$ Centro Nacional de Investigaciones Cardiovasculares, Madrid (Spain)

${ }^{4}$ UC Davis MIND Institute, University of California Davis, Sacramento, CA (US)

${ }^{5}$ Department of Pediatrics, University of California Davis, Sacramento, CA (US)

${ }^{6}$ Department of Anatomic Pathology, University of California San Francisco, San Francisco, CA (US)

${ }^{7}$ Institució Catalana de Recerca i Estudis Avançats (ICREA), Barcelona (Spain)

${ }^{8}$ Department of Biochemistry and Molecular Pharmacology, New York University Grossman School of Medicine, New York, NY (US)

* To whom correspondence should be addressed:

Pau Castel, PhD

Department of Biochemistry and Molecular Pharmacology

New York University Grossman School of Medicine

430 East $29^{\text {th }}$ Street, ACLS East, Room 828

New York, NY 10016

$\mathrm{Ph}:+1$ 212-263-6234

pau.castel@nyulangone.org

\section{Keywords}

LZTR1, RIT1, RAS, Ric, Ras85D, CG3711, RASopathy, Noonan syndrome 


\section{Abstract}

42

43 RAS GTPases are highly conserved proteins involved in the regulation of mitogenic

44 signaling. We have previously described a novel Cullin 3 RING E3 ubiquitin ligase

45 complex formed by the substrate adaptor protein LZTR1 that binds, ubiquitinates, and

46 promotes proteasomal degradation of the RAS GTPase RIT1. In addition, others have

47 described that this complex is also responsible for the ubiquitination of canonical RAS

48 GTPases. Here, we have analyzed the phenotypes of LZTR1 loss-of-function mutants in

49 both fruit flies and mice and have demonstrated biochemical dependency on their RIT1

50 orthologs. Moreover, we show that LZTR1 is haplosufficient in mice and that embryonic

51 lethality of the homozygous null allele can be rescued by deletion of RIT1.

52

53

54

55

56

57

58

59

60

61

62

63

64

65

66

67

68

69

70

71

72

73

74

75

76

77

78

79

80 


\section{Introduction}

Dysregulation of signal transduction by the RAS family of guanosine 5'-triphosphate (GTP) hydrolases (GTPases) can have profound effects on human development and cause genetic disorders collectively termed RASopathies (Castel et al. 2020; Rauen 2013). Ras GTPases exhibit high affinity towards guanine nucleotides and act as molecular switches by mediating GTP hydrolysis. The nucleotide cycling of RAS GTPases is tightly regulated by GTPase activating proteins (GAPs) (e.g. neurofibromin) and guanine nucleotide exchange factors (GEFs) (e.g. SOS1) that facilitate nucleotide hydrolysis or loading, respectively (Simanshu et al. 2017). Upon GTP binding, RAS proteins undergo a conformational change, which promotes the interaction with different protein effectors that activate downstream signaling pathways, including Raf/MEK/ERK mitogen activated protein kinase (MAPK) and phosphoinositide 3-kinase (PI3K) pathways. Although GAPs and GEFs can rapidly affect the nucleotide cycling of RAS proteins, and hence their activity, other accessory proteins can modulate downstream signaling by regulating the stability and/or activity of RAS GTPases.

Noonan syndrome (NS) is a common RASopathy that is characterized by craniofacial dysmorphism, short stature, congenital heart disease, and developmental delays. The cause of NS has been linked to genetic alterations that result in hyperactivation of the Raf/MEK/ERK MAPK pathway, including recently reported gain-of-function mutations in the RAS GTPase RIT1 and loss-of-function mutations in the BTB protein LZTR1 (Aoki et al. 2013; Yamamoto et al. 2015; Johnston et al. 2018). We have previously reported that RIT1 is mostly bound to GTP in cells, suggesting that it either lacks a GAP, uses a less active GAP, or relies on its intrinsic GTPase activity (Castel et al. 2019). An alternative regulatory mechanism of RIT1 activity is through protein degradation; we identified a Cullin-3 RING E3 ubiquitin ligase complex (CRL3) that uses LZTR1 as a substrate receptor (CRL3 ${ }^{\text {LZTR1 }}$ ) to bind RIT1 and promote its ubiquitination and proteasomal degradation. CRL ${ }^{\text {LZTR1 }}$ preferentially binds to GDP-bound RIT1 and maintains tight regulation of RIT1 protein levels. Importantly, NS-associated RIT1 and LZTR1 missense

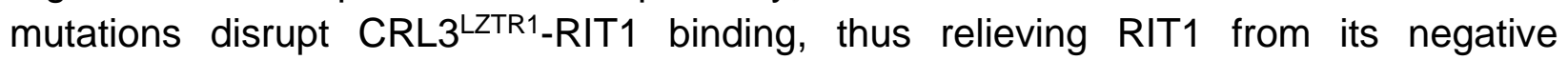
regulatory mechanism.

LZTR1 has been reported to regulate the protein stability of other RAS GTPases by similar mechanisms (Bigenzahn et al. 2018; Steklov et al. 2018; Abe et al. 2020). In this study, we employed a molecular evolutionary approach to elucidate the functional relationship and co-evolution of LZTR1 and its cognate RAS GTPase substrate(s), including the evaluation of LZTR1 loss-of-function mutations in invertebrate and mammalian model organisms. 
The RAS family of GTPase proteins has been previously shown to be well-conserved across species and LZTR1 has been proposed to regulate a subset of these proteins (Rojas et al. 2012). Therefore, to understand how LZTR1 has evolved in comparison with other RAS GTPases, we performed protein alignment analysis of the human orthologs of KRAS, RIT1, and LZTR1 orthologs from human and common model organisms. Consistent with previous studies, KRAS was highly conserved in less complex organisms, including fission yeast (Ras1; 60\% protein identity) (Figure 1A). In contrast, we found that RIT1 rapidly diverged in less complex organisms and a mildly conserved protein in Drosophila melanogaster (RIC; 60\% protein identity) was the most distant ortholog that could be found in these laboratory model species. LZTR1 followed a similar pattern as RIT1; we could identify an ortholog in fruit flies (CG3711/LZTR1; 60\% protein identity), but not in the roundworm Caenorhabditis elegans (Figure 1A). These analyses suggest that RIT1 and LZTR1 are more likely to have functionally co-evolved than KRAS and LZTR1. To further assess the biochemical relationship between these proteins across model organisms, we generated recombinant proteins for the KRAS, RIT1, and LZTR1 orthologs from mouse (Mus musculus), zebrafish (Danio rerio), and fruit fly (Drosophila melanogaster) and tested their ability to interact in pull down assays. All LZTR1 orthologs preferentially interacted with RIT1 orthologs (Figure 1B), indicating that this interaction that we previously reported for human proteins is also conserved in less complex model organisms.

To better understand the role of the LZTR1 ortholog in Drosophila melanogaster, we isolated Lztr1 loss-of-function mutations by using two independent CRISPR/Cas9 gene editing approaches. One resulting allele, $L z t r 1^{1}$, causes a frameshift at the beginning of the second exon and encodes a short, truncated product. Another allele, Lztr $1^{2}$, lacks all coding sequences and is, therefore, a null mutation (Figure 2A). Lztr1 null flies were normal and fertile and did not display any obvious phenotype. A previous study showed that flies expressing RNA interference constructs against Lztr1 displayed minor defects in wing vein patterning (Bigenzahn et al. 2018); however, we did not find consistent vein defects in our null mutants (Figure 2B). head extracts from $L z t r 1^{2}$ and background matched control $(y w)$ flies. Ric levels were upregulated $\sim 11$ times in $L z t r 1^{2}$ fly heads relative to the control, while Ras85D (the HRAS, KRAS, and NRAS ortholog, hereafter referred to as Ras) levels were only modestly

158 altered in both backgrounds ( 1.5-fold difference) (Figure 2C). Similarly, Ras64D (the 159 MRAS ortholog) was only upregulated $\sim 1.6$-fold in $L z t r 1^{2}$ mutant heads. To validate these results, we generated Drosophila transgenic lines carrying N-terminally HA tagged Ric or 
161 Ras genes under the control of their natural genomic sequences, thereby facilitating detection of both products at their endogenous levels. Both transgenes were then placed in the $L z t r 1^{2}$ mutant background to assess the effect of Lztr1 depletion on Ric and Ras protein stability. Consistent with our mass spectrometry results, HA-Ric levels were elevated in the absence of Lztr1, while HA-Ras levels remained barely altered in either mutant adult flies or third-instar larvae (Figure 2D-E). Altogether, these experiments show that Drosophila Lztr1 preferentially regulates Ric rather than Ras levels, as seen with the corresponding mammalian orthologs.

Given the absence of a phenotype in fruit flies, we examined the effect of $L z \operatorname{tr} 1$ deletion in mice. Steklov et al had previously reported that Lztr1 heterozygous knockout mice display typical features of NS, suggestive of haploinsufficiency (Steklov et al. 2018). In humans, NS-associated variants are most commonly found as bi-allelic loss-of-function variants that are inherited in an autosomal recessive manner; although there are few heterozygous variants that segregate as autosomal dominant, these are single nucleotide variants that likely act as dominant negatives (Johnston et al. 2018; Yamamoto et al. 2015; Motta et al. 2019). Hence, we further analyzed the potential NS-like phenotype of heterozygous Lztr1 deletion in the mouse as previously described in other mouse models (Araki et al. 2004; Wu et al. 2011; Hernández-Porras et al. 2014; Castel et al. 2019). Heterozygous mice did not exhibit significant changes in body weight and, overall, their morphology of the skull, which generally shows dysmorphic features in mouse models of NS (round skull, blunt snout, and hypertelorism), was assessed using micro-computed tomography $(\mu \mathrm{CT})$ and did not have significant differences when compared to wild type littermates (Figure 3B). In addition, we assessed whether heterozygous mice displayed either cardiomegaly or splenomegaly, as these signs have been observed in other NSlike mouse models, but we failed to observe differences with wild type animals (Figure 3C-D). These results indicate that Lztr1 heterozygous mice do not exhibit the typical features seen in other NS mouse models and indicate that Lztr1 is haplosufficient for this phenotype. In support of these observations in mice, NS families carrying LZTR1 alleles generally exhibit an autosomal recessive inheritance pattern and bi-allelic inactivation of LZTR1 is required to exhibit a NS phenotype (Johnston et al. 2018). In addition, analysis of LZTR1 single nucleotide variants in the genome aggregation database (gnomAD) shows that more loss-of-function mutations are observed in this gene than expected in healthy individuals ( $\mathrm{pLI}=0$; o/e=2.28) (Karczewski et al. 2020) (Figure 3 - supplement 2). This indicates that these heterozygous loss-of-function variants are tolerated and is highly suggestive of haplosufficiency in humans.

We and others have previously shown that Lztr1 knockout mice are embryonically lethal (Steklov et al. 2018; Castel et al. 2019); however, the reason for such lethality remains 
201 largely unknown. A previous study showed that Lztr1-associated embryonic lethality can

202 be rescued by administering a MEK1/2 inhibitor to pregnant females, indicating that the 203 phenotype is largely dependent on MAPK hyperactivation (Steklov et al. 2018). In fact, in 204 other murine NS models, excessive activation of MAPK during embryonic development 205 results in lethality, as seen in the Kras ${ }^{\mathrm{V} 14 \mathrm{I}}$ model (Hernández-Porras et al. 2014). 206 Interestingly, this phenotype appears to be more prominent in certain mouse background 207 strains, such as C57BL/6N, and more permissive in 129Sv (Araki et al. 2004; Hernández208 Porras et al. 2015). Since our Lztr1 mutant mice are in the C57BL/6N strain, we 209 hypothesized that mouse background affects embryonic lethality. Backcrossing our mice 210 to $129 S v$ females yielded heterozygous Lztr1 mutant mice in a 50\% mixed background. 211 After one backcross (referred to as $\mathrm{F} 1$ ), using a heterozygous $\mathrm{x}$ heterozygous breeding 212 scheme, we obtained 1/55 ( 1.8\%) homozygous viable mice. At F2, we obtained 3/45 $213(\sim 6.7 \%)$ homozygous viable mice. At F3, we obtained $3 / 34$ ( 8.8\%) homozygous viable 214 mice (Figure 3E). In contrast, mice in the 129Sv pure background did not yield any 215 homozygous viable mice, suggesting that a combination of strain-specific genetic 216 modifiers is likely required to tolerate Lztr1 deletion. In Raf1 D486N NS mice, a potential 217 gene modifier was mapped at chromosome 8 of the 129Sv strain (Wu et al. 2012); 218 however, we hypothesize that in the context of Lztr1, a negative gene modifier is also 219 likely to be located in the $\mathrm{Y}$ chromosome of the 129Sv strain, given that we do not yield 220 any homozygous viable mice after Y-chromosome fixing.

221 The limited number of viable homozygous Lztr1 knockout mice prevented us from 222 undertaking quantitative phenotyping; however, when compared to wild type littermates, 223 Lztr1 knockout mice appeared smaller, displayed characteristic dysmorphic facial 224 features (round snout, hypertelorism, low set of ears, and proptosis), and exhibited 225 cardiomegaly, consistent with other mouse models of NS (Figure 3F). In addition, Lztr1 knockout mice exhibited increased levels of Rit1 in all the tissues that we analyzed, while RAS levels remained mostly unchanged (Figure $\mathbf{3 G}$ ).

Next, we sought to investigate the cause of embryonic lethality in Lztr1 knockout mice. We and others had previously shown that many embryos survive to late developmental stages (i.e. embryonic day (E)17.5-19.5), therefore, we harvested embryos on day E18.5. Most embryos display extensive hemorrhages (Figure 4A) and, consistent with this observation, conditional deletion of Lztr1 in blood vessels has been previously shown to cause vascular leakage (Sewduth et al. 2020). Since many NS alleles that cause lethality in mice have been related to cardiovascular dysfunction, we analyzed the heart phenotype of E18.5 embryos. Valve leaflets and endocardial cushions were normal, in contrast to the previous Ptpn11 NS-associated alleles (Araki et al. 2004). In Lztr1 knockout embryos, the ventricular myocardial wall showed defects that are compatible with ventricular noncompaction cardiomyopathy, similar to that seen in the Ptpn11 Q79R transgenic mouse model (Nakamura et al. 2007). Detailed analysis of compact and 
241 trabecular myocardial thickness showed significant differences in mutant embryos 242 (Figure 4B). In addition, we observed interventricular septal defects in 20\% mutant 243 hearts. Although the heart phenotype is striking, it is likely that embryonic lethality results 244 from a combination of vascular and cardiac defects during development.

Lztr1 embryonic lethality provides a unique model to undertake a genetic epistatic rescue experiment to assess which RAS GTPase is the critical downstream substrate of the CRL3 ${ }^{\text {LZTR1 }}$ complex. We have previously shown that at the biochemical level CRL3 ${ }^{\text {LZTR1 }}$ complex preferentially binds and promotes degradation of RIT1. Therefore, we generated Rit1 knockout mice to test whether Rit1 depletion can rescue Lztr1-mediated embryonic lethality. To avoid potential heterosis, we generated Rit1 knockouts in the C57BL6/N background; mice were born at expected Mendelian ratios, were fertile, and did not exhibit any visible phenotype, similar to previous Rit1 null mouse strains (Cai et al. 2011). We confirmed the elimination of Rit1 by immunoblot in different tissues, including brain, lung, and heart (Figure 4C). Next, we crossed Rit1 knockout mice with Lztr1 heterozygous mice to obtain both $L z t r 1^{-/+} / R i t 1^{+/+}$and $L z t r 1^{-1+} / R i t 1^{-/-}$progeny. As previously shown, a heterozygous breeding scheme with $L z t r 1^{-/+} / R i t 1^{+/+}$mice did not yield any viable Lztr1 knockout mice (Figure 3E, 4D) (Sewduth et al. 2020). In contrast, when Lztr1 ${ }^{-/+} /$Rit $^{-/-}$ breeders were used, we obtained 13/86 ( 15.1\%) mice that were double knockout (DKO) for both Lztr1 and Rit1 (Figure 4D). This result shows that Rit1 deletion can rescue the embryonic lethality caused by Lztr1 deletion in mice, indicating that Rit1 is the critical substrate of the CRL3 ${ }^{\text {LZTR1 }}$ complex during embryonic development. The resulting DKO mice appeared normal, were fertile, and were absent of any detectable phenotype that resembled other NS mouse models, as assessed by size, heart weight, and cranial morphology. In addition, Lztr1/Rit1 DKO embryos harvested at E18.5 showed a complete rescue of the cardiac phenotype, which were indistinguishable from Rit1 KO embryos (Figure 4E). To assess whether the Rit1 dependency established by Lztr1/Rit1 DKO mice is correlated to a rescue of MAPK pathway hyperactivation, we isolated primary mouse embryonic fibroblasts (MEF) from wild type, Lztr1 KO, Rit1 KO, and Lztr1/Rit1 DKO embryos (Figure 4F). We then subjected these MEFs to FBS stimulation and observed a noticeable decrease in MAPK signaling in cells devoid of both LZTR1 and RIT1 compared to LZTR1 KO cells, indicating that MAPK pathway hyperactivation in Lztr1 mutant cells is mediated by RIT1 protein stabilization in the absence of CRL3 ${ }^{\text {LZTR1 }}$ regulation (Figure $\mathbf{4 G}$ ).

Many human RAS proteins are highly conserved in less complex organisms and a common ancestor Ras protein has been described in distant relatives such as yeast and slime mold, among other Eukarya (Cox and Der 2010). Similarly, certain regulators of RAS function, such as GAPs or GEFs are also conserved in these organisms (van Dam et al. 2011). Here, we show that in contrast to the highly conserved protein KRAS, both 
RIT1 and LZTR1 proteins are less conserved in lower organisms. Despite this, LZTR1 orthologs of invertebrate model organisms retain preferential binding towards their respective RIT1, but not RAS, orthologs. Moreover, fruit flies exhibit strong conservation of the LZTR1-mediated protein degradation mechanism with specificity towards RIT1, as we had previously described with human orthologs, but minimal activity towards other fruit fly RAS GTPases. These data indicate a functional co-evolution between LZTR1 and RIT1.

We also demonstrate that, in contrast to previous reports, LZTR1 is haplosufficient in mice (Steklov et al. 2018). However, homozygous knockout mutations are not tolerated due to embryonic lethality caused by aberrant cardiovascular development concomitant with severe peripheral hemorrhaging and ventricular septal defects, which can be rescued by Rit1 germline deletion. Interestingly, Sewduth et al., demonstrate that pharmacological inhibition of the RAF/MEK/ERK MAPK or AKT pathway was unable to fully rescue the embryonic lethality and vascular defects of mice with conditional Lztr1 KO in blood vessels (Sewduth et al. 2020). Given that our Lztr1/Rit1 DKO mice did not exhibit cardiovascular abnormalities during embryogenesis, excessive accumulation of Rit1 protein in Lztr1 $\mathrm{KO}$ cells likely results in the hyperactivation or dysregulation of additional Rit1 effector pathways that contribute to $\mathrm{Lztr}^{-1-}$ embryonic lethality (Cuevas-Navarro et al. 2021).

While other RAS GTPases have cognate GAPs and GEFs that regulate their activity, RIT1 GAPs and GEFs remain to be identified. In light of our findings, we posit that LZTR1 may have co-evolved with RIT1 as an alternative mechanism to regulate RIT1 GTPase activity. 


\section{Materials and methods}

\section{DNA constructs}

Human, mouse (Mus musculus), zebrafish (Danio rerio) and fruit fly (Drosophila melanogaster) RIT1 and KRAS orthologs were synthesized as E. coli codon-optimized gene blocks (IDT) and cloned into pDONR221. Gateway LR reaction was used to generate a pGEX-6P destination vector for bacterial expression. Human, mouse (Mus musculus), zebrafish (Danio rerio) and fruit fly (Drosophila melanogaster) LZTR1 orthologs were synthesized as $H$. sapiens codon-optimized gene blocks (IDT) and cloned into pDONR221. Gateway LR reaction was used to generate a pcDNA3-HA-tagged destination vector for mammalian expression. All plasmids were verified by Sanger sequencing.

\section{Recombinant proteins}

GST-tagged recombinant proteins were expressed in BL21 (DE3) E. coli cells and expression was induced with $0.2 \mathrm{mM}$ IPTG for $14-16 \mathrm{~h}$ at $18^{\circ} \mathrm{C}$. Cells were lysed by sonication in $50 \mathrm{mM}$ Tris- $\mathrm{HCl}$ (pH 8.0), $300 \mathrm{mM} \mathrm{NaCl}, 5 \%$ glycerol, $1 \mathrm{mM}$ DTT. Proteins were immobilized on Glutathione Sepharose 4B beads (Cytiva Life Sciences), washed extensively, and stored as a $50 \%$ glycerol bead suspension at $-20^{\circ} \mathrm{C}$. HA-tagged recombinant proteins were expressed in HEK-293T cells by transient transfection and collected in lysates after 24 hours.

\section{Immunoblot}

344 Protein samples were prepared from frozen mouse tissues using RIPA buffer and a Dounce tissue homogenizer. Drosophila samples were prepared from either frozen L3 instar larvae, adults, or isolated fly heads and were lysed in RIPA lysis buffer with a Dounce homogenizer. Protein lysates were cleared by centrifugation. For immunoblot detection, samples were separated by SDS-PAGE (NuPAGE) and transferred onto nitrocellulose membranes using iBlot2. Membranes were blocked using $2.5 \%$ skimmed milk in TBS-T buffer for 1 hour at room temperature and incubated with appropriate primary antibodies overnight. Detection was performed using HRP-linked secondary antibodies and developed with Amersham ECL (Cytiva Life Sciences) and X-ray films. Antibodies used in this work were: HA (Cell Signaling Technologies, Cat \#3724; 1:3,000), B-Actin (Sigma-Aldrich, Cat \#A2228; 1:10,000), a-Tubulin (Sigma-Aldrich, Cat \#T6199; 1:10,000), LZTR1 (Santa Cruz Biotechnology, Cat \#sc-390166X; 1:1,000), RIT1 (Abcam, Cat \#ab53720; 1:1,000), p-ERK (Cell Signaling Technologies, Cat \#4370; 1:1000), ERK1/2 (Cell Signaling Technologies, Cat \#4696; 1:2000), p-MEK (Cell Signaling $1: 1000)$. 


\section{Mice}

362 The Lztr1 allele (Lztr1 1m1a(EUCOMM)Wtsi) was previously described. Briefly, frozen sperm was 363 obtained from the Knockout Mouse Project and IVF was performed on C57BL/6NTac 364 eggs. Heterozygous mice were maintained in C57BL/6NTac congenic background. Every 365 six months, new C57BL/6NTac males (Taconic) were introduced to our colony to avoid genetic drift. Homozygous knockout Lztr1 embryos (E18.5) were obtained from timed pregnancies using a heterozygous $x$ heterozygous breeding scheme. 129S1/Svimj mice were purchased at the Jackson Laboratories. Mixed C56BL/6N-129S1 mice were obtained by crossing Lztr1 heterozygous male C56BL/6N mice with $129 \mathrm{~S} 1$ females for the first 5 back crossings. Then,129S1 males were introduced for Y-chromosome fixing. Knockout Lztr1 mice were considered to be in a pure 129S1 background after at least eight backcrosses. Rit1 knockout mice were generated by Cyagen using CRISP/Cas9mediated large homology arm recombination in C57BL/6NTac fertilized eggs. Briefly, a cassette containing a loxP-STOP-loxP was engineered to be located at the first coding exon of Rit 1 and was microinjected with appropriate gRNA and Cas9 mRNA into zygotes and transferred into the oviducts of pseudopregnant females. Mice with germline transmission of the Rit1 allele were used as founders to establish the Rit1 knockout homozygous colony. This study was performed in strict accordance with the recommendations in the Guide for the Care and Use of Laboratory Animals of the National Institutes of Health. All of the animals were handled according to approved institutional animal care and use committee (IACUC) protocols (\#AN165444 and \#AN179937) of the University of California San Francisco.

\section{Mouse phenotyping}

Noonan syndrome-like phenotypes in mice were analyzed as previously reported (Castel et al. 2019). Briefly, mice were weaned at 3 weeks of age, weighted at 4 weeks, skull CT was performed at 8 weeks and then mice were euthanized for heart measurements. Sample size was calculated based on anticipated values from our previous phenotyping experiments using a Rit ${ }^{\text {M90l }}$ Noonan syndrome mouse model (Castel et al. 2019). For skull morphometry, sample size was determined to be at least $n=5$ to achieve an alpha of 0.05 with a power of $80 \%$. For body weight, sample size was determined to be at least $n=13$, and for organ-to-body weight measurements, sample size was determined to be at least $n=6$, following the same statistical parameters described above.

For E18.5 embryos, trunks were fixed overnight in $10 \%$ buffered formalin, dehydrated with ethanol, and embedded in paraffin. For cardiac analysis, the whole trunk was sectioned transversely at $6 \mu \mathrm{m}$ and $\mathrm{H} \& \mathrm{E}$ staining was performed following standard protocols at the Histology Core Facility of the National Center for Cardiovascular Research (CNIC). Ventricular wall measurements were obtained from H\&E-stained sections. The length of at least 4 lines from a minimum of 8 sections obtained from 3 
401 different embryos were measured with Ruler tool (NDP.View 2 Software). Statistical 402 analyses were carried out using Prism 7 (GraphPad). Data are presented as mean \pm SEM 403 unless stated otherwise. Statistical significance was determined by performing 2-tailed, 404 unpaired Student's $t$ tests when comparing 2 groups. $P$ values of less than 0.05 are 405 considered significant. At least three independent dissections were performed to obtain 406 E18.5 embryos.

\section{Drosophila strains and crosses}

409 Flies were maintained on standard food medium under a 12:12 light:dark cycle at $25^{\circ} \mathrm{C}$. The Lztr1 ${ }^{1}$ allele was generated by CRISPR-Cas9-mediated mutagenesis using transgenic nanos-Cas9 (Kondo and Ueda 2013; Ren et al. 2013) and guide RNA (gRNA) lines. Briefly, a double gRNA expression construct directed against the following protospacer sequences, 5'

GAAGCAAGCACACAGTGG-3'

and $5^{\prime}-$ GATGCGATGTTTGTATTCGG-3' (both corresponding to the upper strand), was generated in vector pBFv-U6.2B (Kondo and Ueda 2013) and inserted via ФC31 integrase-mediated transformation at the attP40 landing site (Bischof et al. 2007). The resulting line was then crossed to nanos-Cas9-expressing flies to isolate Lztr1 mutations, including Lztr11. Lztr12 ${ }^{2}$ was obtained via CRISPR-Cas9 engineering by GenetiVision Corporation and verified by PCR amplification and sequencing. HA-tagged Ric- and Rasexpressing constructs were ordered as custom genes from GenScript and transferred into the pattB vector (Bischof et al. 2013). Both contain the corresponding Ric and Ras genomic sequences including their respective 5' and 3' regulatory regions, with the HA coding sequence inserted after the initiation codon. Transgenic lines for each construct were established by $\Phi \mathrm{C} 31$-mediated integration at the attP-86Fb landing site. Both homozygous lines are viable and fertile and were placed in the $L z t r{ }^{2}$ mutant background by standard crosses.

\section{Quantification of protein abundance by mass spectrometry}

428 Drosophila head lysates ( $300 \mu \mathrm{g}$ in $100 \mu \mathrm{L}$ per sample) were precipitated with acetone 429 by adding $400 \mu \mathrm{L}$ of ice-cold acetone, vortexing the lysates briefly, incubating the lysates 430 at $-20^{\circ} \mathrm{C}$ for $1 \mathrm{hr}$, centrifuging at $15,000 \times \mathrm{g}$ for 10 minutes and decanting the 431 supernatants. The protein pallets were then dissolved in $20 \mu \mathrm{L}$ of $50 \mathrm{mM} \mathrm{Tris-HCl}$ buffer, $432 \mathrm{pH} 8.0$ containing $8 \mathrm{M}$ urea with vortexing. The samples were reduced by adding $1.2 \mu \mathrm{L}$ 433 of $100 \mathrm{~mm}$ dithiothreitol and vortexing for $30 \mathrm{~min}$ at room temperature (RT), followed by 434 alkylation with added $3.5 \mu \mathrm{L}$ of $100 \mathrm{mM}$ iodoacetamide and gentle vortexing for $30 \mathrm{~min}$ in 435 the dark for 30 min at RT. One $\mu \mathrm{L}$ from each sample was used to quantify protein amounts 436 in using BCA protein assay kit (Thermo Fisher Scientific, USA) according to the 437 manufacturer's protocol. The samples were diluted by adding $90 \mu \mathrm{L}$ of $50 \mathrm{mM} \mathrm{Tris-} \mathrm{HCl}$ 438 buffer, $\mathrm{pH} 8.0$ containing $1 \mathrm{mM} \mathrm{CaCl} 2$ and digested with $6 \mathrm{ug}$ trypsin overnight at $37^{\circ} \mathrm{C}$ 439 with agitation. The samples were then acidified by adding $1 \mu \mathrm{L}$ of formic acid, and the 
440 digested peptides were desalted using Sep-Pak C18 Classic Cartridges (Waters, USA) 441 using the manufacturer's protocol.

For LC-MS/MS analysis, digested and desalted peptides were suspended in $0.1 \%$ formic acid, and $0.5 \mathrm{ug}$ was injected per sample on the EasySpray $50 \mathrm{~cm}$ C18 column (ES903, Thermo Fisher Scientific, USA) using Acquity UPLC M-Class System (Waters, USA) on line with Orbitrap Fusion Lumos Tribrid Mass Spectrometer (Thermo Fisher Scientific, USA). The column was held at $45^{\circ} \mathrm{C}$, and a $185-\mathrm{min}$ low-pH reversed phase chromatography linear gradient was performed using conventional two-buffer system (buffer A: $0.1 \%$ formic acid in MS-grade water; buffer B: $0.1 \%$ formic acid in acetonitrile) by increasing buffer B concentration from 3.5\% to $30 \%$ over $185 \mathrm{~min}$, followed by a 2 min wash to $50 \%$, with a constant flow rate of $300 \mathrm{~nL} / \mathrm{min}$. The mass spectrometer was operated in positive ionization mode with the spray voltage of $2.5 \mathrm{KV}$, ion transfer tube temperature of $275^{\circ} \mathrm{C}, \mathrm{RF}$ lens at $30 \%$, and internal calibration set to Easy-IC. MS1 spectra were collected in the Orbitrap in profile mode at $120 \mathrm{~K}$ resolution, $375-1500 \mathrm{~m} / \mathrm{z}$ mass range, 50 ms maximum injection time (IT), and automatic gain control (AGC) target of 4.0e5. Precursor ions charged $2+$ to $7+$ with MS1 intensity above the threshold of 2.0e4 were isolated in the Quadruple using 1.6 Th isolation window, fragmented using higher-energy collisional dissociation (HCD) with $30 \%$ collision energy, and detected in the Orbitrap in Centroid mode at $30 \mathrm{~K}$ resolution, scan range set to Auto Normal, $100 \mathrm{~ms}$ maximum IT, and 5.0e4 AGC target. The maximum cycle time was set to $3 \mathrm{~s}$ and dynamic exclusion to $30 \mathrm{~s}$ with $+/-10 \mathrm{ppm}$ tolerance.

MS .raw data files were converted to peak lists using PAVA in-house script and searched with Protein Prospector (v 6.0.0) (Chalkley et al. 2008) against Drosophila UniProtKB FASTA-formatted database, which included proteins and their splicing isoforms downloaded on 2019-04-19 (uniprot.org) and a corresponding random-concatenated database of decoy peptides added with Protein Prospector. Instrument was set to "ESIQ-high-res." Enzyme was set to Trypsin. Up to 2 missed cleavages were allowed and up to 2 post-translational modifications per peptide. "Carbamidomethyl (C)" was set as a constant modification, and default variable modifications were allowed: Acetyl (Protein N-term), Acetyl+Oxidation (Protein N-term M), Gln->pyro-Glu (N-term Q), Met-loss (Protein N-term M), Met-loss+Acetyl (Protein N-term M), Oxidation (M), and Oxidation (P). Precursor charges were set to $2+$ to $5+$, MS1 mass tolerance to $10 \mathrm{ppm}$, and MS2 mass tolerance to 20 ppm. FDR filters for peptides was set to $1 \%$ and for proteins to 5\%. The search results were exported from Protein prospector as tab-delimited text and as .blib spectral library, which was imported into Skyline (v20) along with .raw files for quantification using MS1 filtering (Schilling et al. 2012). The identified peaks were detected by training built-in mProphet model against corresponding random-sequence decoy peptides (Reiter et al. 2011), peaks with an FDR $<1 \%$ were integrated. Built-in 
MSstats (v 3.13) (Choi et al. 2014) plugin in Skyline was used to normalize runs by median centering log2 precursor intensities, to calculate aggregate protein abundances, and to estimate their statistical significance.

\section{Competing Interest Statement}

FM is is a consultant for Ideaya Biosciences, Kura Oncology, Leidos Biomedical Research, Pfizer, Daiichi Sankyo, Amgen, PMV Pharma, OPNA-IO, and Quanta Therapeutics and has received research grants from Boehringer-Ingelheim and is a consultant for and cofounder of BridgeBio Pharma. PC is a founder and advisory board of Venthera. The other authors declare no competing interests.

\section{Acknowledgements}

We thank Tony Huynh and Juan Antonio Camara Serrano for help with microCT imaging and Stephanie Mo for feedback on the manuscript. JG-B was funded by Programa "Atracción de Talento" de la Comunidad de Madrid. This work was supported by the $\mathrm{NCl}$ (1F31CA265066 to AC-N), (R35CA197709 to FM), (K99CA245122 to PC) and the Department of Defense Neurofibromatosis Research Program (W81XWH-20-1-0391 to PC). We thank the UCSF Mass Spectrometry Facility and A. L. Burlingame for providing MS instrumentation support for this project (funded by the NIH grants P41GM103481 and S10OD016229). GJ and LR-M were funded by grants from the Spanish Government (BFU2017-87244-P, PID2020-119248GB-I00 and Predoctoral contract BES-2015071486).

\section{Author contributions}

Conceptualization: PC; Methodology: AC-N, LR-M, AU, PC; Investigation: AC-N, LR-M, JG-B, AC, KAR, AU, GJ, FM, PC; Writing: AC-N, PC, and all authors approved final manuscript; Resources: LR-M, GJ, AU; Visualization: PC, Supervision: GJ, FM, PC; 


\section{References}

521

522

523

524

525

526

527

528

529

530

531

532

533

534

535

536

537

538

539

540

541

542

543

544

545

546

547

548

549

550

551

552

553

554
Abe T, Umeki I, Kanno S-I, Inoue S-I, Niihori T, Aoki Y. 2020. LZTR1 facilitates polyubiquitination and degradation of RAS-GTPases. Cell Death Differ 27: 10231035.

Aoki Y, Niihori T, Banjo T, Okamoto N, Mizuno S, Kurosawa K, Ogata T, Takada F, Yano M, Ando T, et al. 2013. Gain-of-function mutations in RIT1 cause Noonan syndrome, a RAS/MAPK pathway syndrome. Am J Hum Genet 93: 173-180.

Araki T, Mohi MG, Ismat FA, Bronson RT, Williams IR, Kutok JL, Yang W, Pao LI, Gilliland DG, Epstein JA, et al. 2004. Mouse model of Noonan syndrome reveals cell type- and gene dosage-dependent effects of Ptpn11 mutation. Nat Med 10: 849-857.

Bigenzahn JW, Collu GM, Kartnig F, Pieraks M, Vladimer GI, Heinz LX, Sedlyarov V, Schischlik F, Fauster A, Rebsamen M, et al. 2018. LZTR1 is a regulator of RAS ubiquitination and signaling. Science 362: 1171-1177.

Bischof J, Björklund M, Furger E, Schertel C, Taipale J, Basler K. 2013. A versatile platform for creating a comprehensive UAS-ORFeome library in Drosophila. Development 140: 2434-2442.

Bischof J, Maeda RK, Hediger M, Karch F, Basler K. 2007. An optimized transgenesis system for Drosophila using germ-line-specific C31 integrases. Proceedings of the National Academy of Sciences 104: 3312-3317.

Cai W, Rudolph JL, Harrison SMW, Jin L, Frantz AL, Harrison DA, Andres DA. 2011. An evolutionarily conserved Rit GTPase-p38 MAPK signaling pathway mediates oxidative stress resistance ed. J. Chernoff. MBoC 22: 3231-3241.

Castel P, Cheng A, Cuevas-Navarro A, Everman DB, Papageorge AG, Simanshu DK, Tankka A, Galeas J, Urisman A, McCormick F. 2019. RIT1 oncoproteins escape LZTR1-mediated proteolysis. Science 363: 1226-1230.

Castel P, Rauen KA, McCormick F. 2020. The duality of human oncoproteins: drivers of cancer and congenital disorders. Nat Rev Cancer 20: 383-397.

Chalkley RJ, Baker PR, Medzihradszky KF, Lynn AJ, Burlingame AL. 2008. In-depth analysis of tandem mass spectrometry data from disparate instrument types. $\mathrm{Mol}$ Cell Proteomics 7: 2386-2398.

Choi M, Chang C-Y, Clough T, Broudy D, Killeen T, MacLean B, Vitek O. 2014. MSstats: an $\mathrm{R}$ package for statistical analysis of quantitative mass spectrometrybased proteomic experiments. Bioinformatics 30: 2524-2526.

Cox AD, Der CJ. 2010. Ras history: The saga continues. Small GTPases 1: 2-27. 
Cuevas-Navarro A, Van R, Cheng A, Urisman A, Castel P, McCormick F. 2021. The RAS GTPase RIT1 compromises mitotic fidelity through spindle assembly checkpoint suppression. Curr Biol 31: 3915-3924.e9.

Hernández-Porras I, Fabbiano S, Schuhmacher AJ, Aicher A, Cañamero M, Cámara JA, Cussó L, Desco M, Heeschen C, Mulero F, et al. 2014. K-Ras V141 recapitulates Noonan syndrome in mice. Proc Natl Acad Sci USA 111: 1639516400.

Hernández-Porras I, Jiménez-Catalán B, Schuhmacher AJ, Guerra C. 2015. The impact of the genetic background in the Noonan syndrome phenotype induced by KRas(V14I). Rare Dis 3: e1045169.

Johnston JJ, van der Smagt JJ, Rosenfeld JA, Pagnamenta AT, Alswaid A, Baker EH, Blair E, Borck G, Brinkmann J, Craigen W, et al. 2018. Autosomal recessive Noonan syndrome associated with biallelic LZTR1 variants. Genet Med 20: $1175-1185$.

Karczewski KJ, Francioli LC, Tiao G, Cummings BB, Alföldi J, Wang Q, Collins RL, Laricchia KM, Ganna A, Birnbaum DP, et al. 2020. The mutational constraint spectrum quantified from variation in 141,456 humans. Nature 581: 434-443.

Kondo S, Ueda R. 2013. Highly improved gene targeting by germline-specific Cas9 expression in Drosophila. Genetics 195: 715-721.

Motta M, Fidan M, Bellacchio E, Pantaleoni F, Schneider-Heieck K, Coppola S, Borck G, Salviati L, Zenker M, Cirstea IC, et al. 2019. Dominant Noonan syndromecausing LZTR1 mutations specifically affect the Kelch domain substraterecognition surface and enhance RAS-MAPK signaling. Hum Mol Genet 28: 1007-1022.

Nakamura T, Colbert M, Krenz M, Molkentin JD, Hahn HS, Dorn GW, Robbins J. 2007. Mediating ERK 1/2 signaling rescues congenital heart defects in a mouse model of Noonan syndrome. J Clin Invest 117: 2123-2132.

Rauen KA. 2013. The RASopathies. Annu Rev Genomics Hum Genet 14: 355-369.

Reiter L, Rinner O, Picotti P, Hüttenhain R, Beck M, Brusniak M-Y, Hengartner MO, Aebersold R. 2011. mProphet: automated data processing and statistical validation for large-scale SRM experiments. Nat Methods 8: 430-435.

Ren X, Sun J, Housden BE, Hu Y, Roesel C, Lin S, Liu L-P, Yang Z, Mao D, Sun L, et al. 2013. Optimized gene editing technology for Drosophila melanogaster using germ line-specific Cas9. Proc Natl Acad Sci U S A 110: 19012-19017.

Rojas AM, Fuentes G, Rausell A, Valencia A. 2012. The Ras protein superfamily: Evolutionary tree and role of conserved amino acids. Journal of Cell Biology 196: 189-201. 
Schilling B, Rardin MJ, MacLean BX, Zawadzka AM, Frewen BE, Cusack MP, Sorensen DJ, Bereman MS, Jing E, Wu CC, et al. 2012. Platform-independent and labelfree quantitation of proteomic data using MS1 extracted ion chromatograms in skyline: application to protein acetylation and phosphorylation. Mol Cell Proteomics 11: 202-214.

Sewduth RN, Pandolfi S, Steklov M, Sheryazdanova A, Zhao P, Criem N, Baietti MF, Lechat B, Quarck R, Impens F, et al. 2020. The Noonan Syndrome Gene Lztr1 Controls Cardiovascular Function by Regulating Vesicular Trafficking. Circ Res 126: 1379-1393.

Simanshu DK, Nissley DV, McCormick F. 2017. RAS Proteins and Their Regulators in Human Disease. Cell 170: 17-33.

Steklov M, Pandolfi S, Baietti MF, Batiuk A, Carai P, Najm P, Zhang M, Jang H, Renzi $F$, Cai $Y$, et al. 2018. Mutations in LZTR1 drive human disease by dysregulating RAS ubiquitination. Science 362: 1177-1182.

van Dam TJP, Bos JL, Snel B. 2011. Evolution of the Ras-like small GTPases and their regulators. Small GTPases 2: 4-16.

Wu X, Simpson J, Hong JH, Kim K-H, Thavarajah NK, Backx PH, Neel BG, Araki T. 2011. MEK-ERK pathway modulation ameliorates disease phenotypes in a mouse model of Noonan syndrome associated with the Raf1L613V mutation. $J$ Clin Invest 121: 1009-1025.

Wu X, Yin J, Simpson J, Kim K-H, Gu S, Hong JH, Bayliss P, Backx PH, Neel BG, Araki T. 2012. Increased BRAF heterodimerization is the common pathogenic mechanism for noonan syndrome-associated RAF1 mutants. Mol Cell Bio/ 32: 3872-3890.

Yamamoto GL, Aguena M, Gos M, Hung C, Pilch J, Fahiminiya S, Abramowicz A, Cristian I, Buscarilli M, Naslavsky MS, et al. 2015. Rare variants in SOS2 and LZTR1 are associated with Noonan syndrome. J Med Genet 52: 413-421. 

a. Phylogenetic trees of KRAS, RIT1, and LZTR1 orthologs based on multiple protein sequence alignments performed with Clustal Omega (Figure 1 - source data 1). Orthologs were searched in the following model organisms: chimpanzee (Pan troglodytes), pig (Sus scrofa), chicken (Gallus gallus), mouse (Mus musculus), African clawed frog (Xenopus laevis), zebrafish (Danio rerio), fruit fly (Drosophila melanogaster), roundworm (Caenorhabditis elegans), and budding yeast (Saccharomyces cerevisiae). b. Pull down assays using GST-tagged KRAS and RIT1 or their mouse, zebrafish, or fruit fly orthologs produced recombinantly in bacteria. Recombinant proteins were incubated with lysates from HEK-293T cells expressing their corresponding species' HA-tagged LZTR1 ortholog.

a. A schematic representation of the Drosophila CG3711/Lztr1 gene locus is shown. Coding exons are represented in blue. Two CRISPR/Cas9-mediated approaches were used to isolate the $L z t r 1$ loss-of-function alleles, $L z t r 1^{1}$ and $L z t r 1^{2}$. b. Wing vein patterning is not affected in Lztr1 null flies (upper panel), with very few individuals exhibiting small ectopic veinlets in two or three points (asterisks; lower panel). c. Estimated normalized protein abundance expressed as mean log2 fold change in $\mathrm{Lztr}^{2}{ }^{2}$ vs yw (control) comparison. Corresponding single-protein standard error (SE) and t-test p-values are listed. d. Immunoblot analysis of protein extracts isolated from the indicated transgenic adult flies in a $y w$ or Lztr1 $^{2}$ background. ns: non-specific band. e. Same as panel d with

Figure 3. Lztr1 is haplosufficient in mice and its null phenotype can be modified by strain background

a. Weight (left panel) and length (right panel) of four-week-old male Lztr1 wild type and heterozygous mutant mice. $\mathbf{b}$. Representative $\mu \mathrm{CT}$ imaging of the skull of an eight-weekold male Lztr1 wild type and heterozygous mutant mouse. The indicated values show the average measurement $(\mathrm{mm})$ of length, width, and inner intercanthal distance in Lztr1 wild type $(n=5)$ and heterozygous mutant $(n=5)$ mice. Mann-Whitney $p$ values were not significant for all the measurements. c. Heart weight was similar between eight-week-old male Lztr1 wild type and heterozygous mutant mice, as assessed by heart to body weight ratio (HW/BW). Mann-Whitney test $p$ value was not significant. d. Same as panel $\mathbf{c}$, for the spleen of these mice. e. Pie charts indicate the percentage of obtained genotypes 
671 upon weaning (21 days of age) the offspring of Lztr1 heterozygous mutant intercrosses.

672 Each pie chart represents a different strain background and/or mixed background. f.

673 Representative image of female littermates with the indicated Lztr1 genotypes

674 (C57BL/6N-129Sv F3 background). Note the decreased size, round skull, and proptosis

675 of the homozygous Lztr1 knockout mouse. g. Immunoblot analysis of RIT1, RAS, and

676 Tubulin proteins isolated from the indicated tissues of $L z \operatorname{tr} 1$ wild type, heterozygous, and

677 homozygous mice (C57BL/6N-129Sv F3 background). Protein lysates from two different

678 mice were used for each genotype.

680 Figure 3 - supplement 1. a. Weight (left panel) and length (right panel) of four-week-old female Lztr1 wild type $(n=13)$ and heterozygous knockout $(n=18)$ mice. b. $\mu C T$

682 measurements of the skull of eight-week-old male and female Lztr1 wild type and heterozygous knockout mice. The indicated values show the average measurement ( $\mathrm{mm}$ ) of length, width, and inner intercanthal distance, as well as the standard deviation (SD). Mann-Whitney $p$ values are indicated and were not significant for any of the measurements. c. Heart weight was similar between eight-week-old female Lztr1 wild type $(n=9)$ and heterozygous mutant $(n=7)$ mice, as assessed by heart to body weight ratio $(\mathrm{HW} / \mathrm{BW})$. Mann-Whitney test $\mathrm{p}$ value was not significant. d. Same as panel $\mathrm{c}$, for the spleen of these mice.

Figure 3 - supplement 2. Snapshot of LZTR1 analysis results obtained from gnomAD v2.1.1 (accessed 12/2021) showing the different variants along the LZTR1 gene. Lossof-function (pLOF) variants are shown in pink. Link: (https://gnomad.broadinstitute.org/gene/ENSG00000099949?dataset=gnomad r2 1)

Figure 4. Lethality in Lztr1 knockout mutants as a result of Rit1-dependent cardiovascular defects E18.5. Note the presence of extensive hemorrhage in knockout embryos (yellow arrows). b. Histological characterization of E18.5 hearts show highly penetrant defects in ventricular wall thickness at both outflow tract (OFT) and atrioventricular canal (AVC) levels, as well as septal defects (arrows) in 20\% Lztr1\% embryos. Quantification of ventricular wall thickness in both $\mathrm{CM}$ (yellow) and TM (green) and CM/TM index are shown in the right graphs $(n=5)$. Rv: right ventricle; Iv: left ventricle; ivs: interventricular septum; CM: compact myocardium; TM: trabecular myocardium. $\mathrm{P}$ values were calculated using Student's t-test. c. Immunoblot analysis of different tissues isolated from

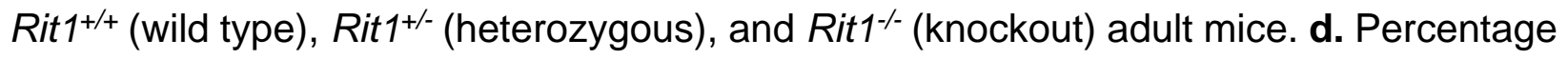
of Lztr1 genotypes obtained upon weaning (21 days of age) the offspring of Lztr1 
711 analysis of the heart of Lztr1; Rit1 double knockout E18.5 embryos. f. Immunoblot 712 analysis of lysates isolated from primary MEF with the indicated genotypes. g. Primary 713 MEF derived from wild type, Lztr1 knockout, Rit1 knockout, and Lztr1; Rit1 double 714 knockout were starved overnight and stimulated with 10\% FBS during the indicated times. 715 Protein lysates were immunoblotted as indicated. 


\section{a}

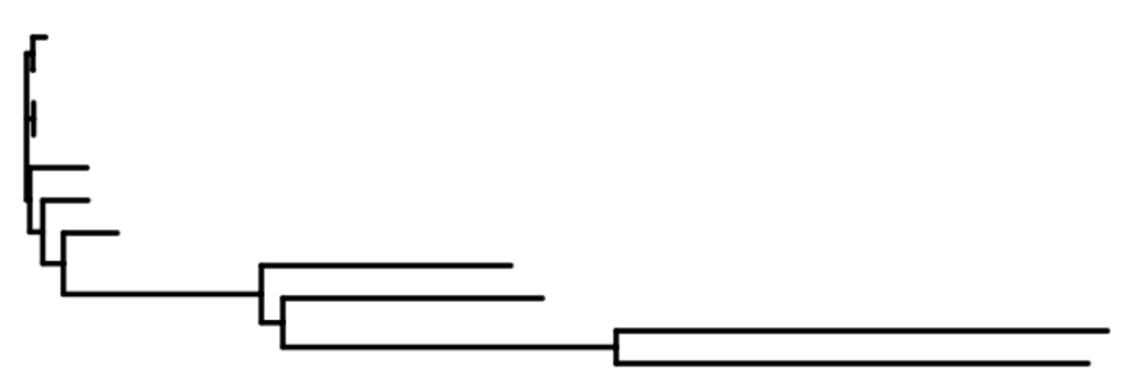

G. gallus Kras

S. scrofa Kras

H. sapiens KRAS

P. troglodytes Kras

$X$. laevis Kras

M. musculus Kras

D. rerio Kras

D. melanogaster Ras85D

C. elegans let-60

S. cerevisiae Ras 1

S. cerevisiae Ras2

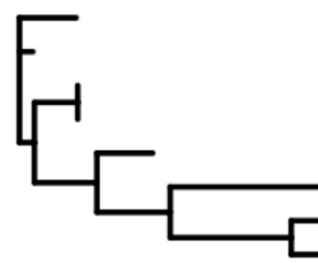

M. musculus Rit1

S. scrofa Rit1

H. sapiens RIT1

P. troglodytes Rit1

G. gallus Rit1

$X$. laevis Rit1

D. melanogaster Ric

D. rerio Rit1

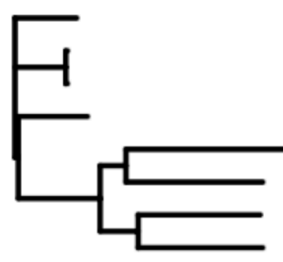

S. scrofa Lztr1

\section{H. sapiens LZTR1}

P. troglodytes Lztr1

M. musculus Lztr1
D. melanogaster CG3711

D. rerio Lztr1

G. gallus Lztr1

$X$. laevis Lztr1

\section{b}

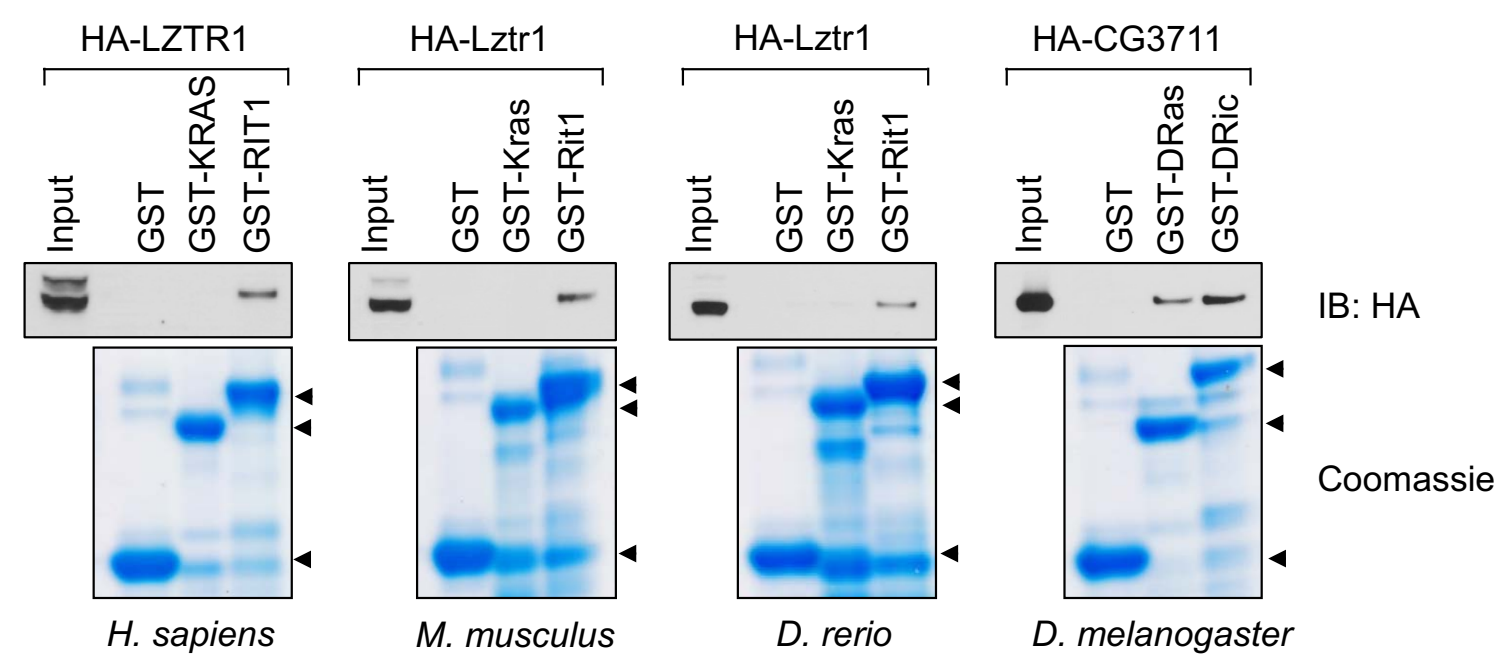


bioRxiv preprint doi: https://doi.org/10.1101/2022.01.04.474894; this version posted January 5, 2022. The copyright holder for this preprint (which was not certified by peer review) is the author/funder, who has granted bioRxiv a license to display the preprint in perpetuity. It is made

a

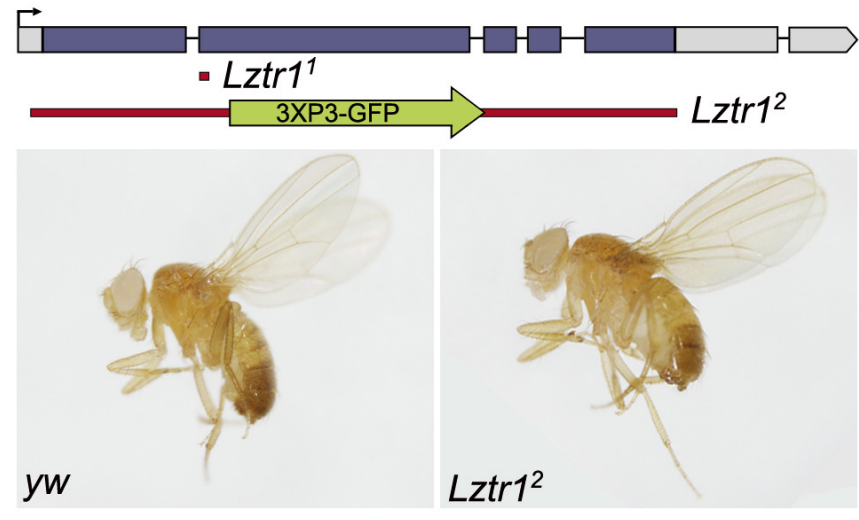

C

\begin{tabular}{ccccc} 
Protein & $\log _{2}$ FC & SE & FC & p-value \\
Ric & 3.474 & 0.217 & 11.12 & 0.003865 \\
Ras85D & 0.611 & 0.150 & 1.53 & 0.055072 \\
Ras64B & 0.724 & 0.191 & 1.65 & 0.06282 \\
\hline
\end{tabular}

d

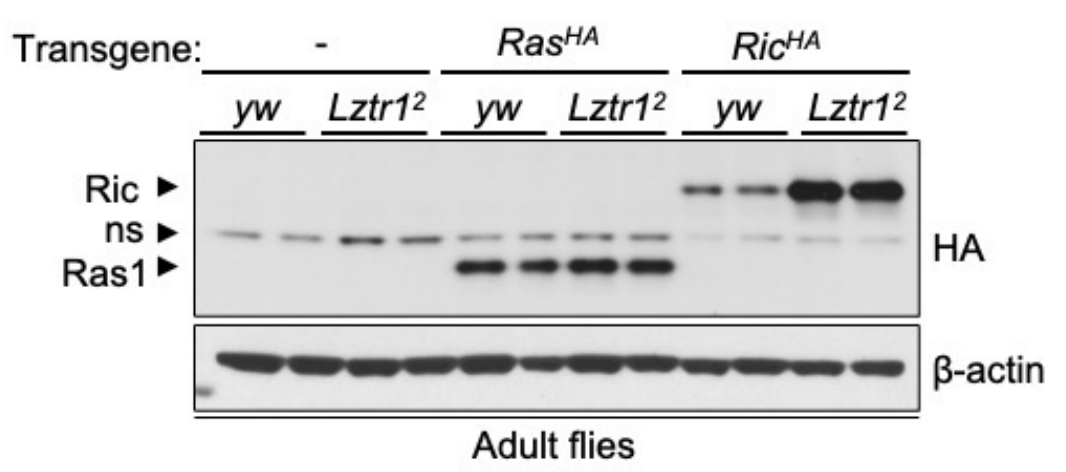

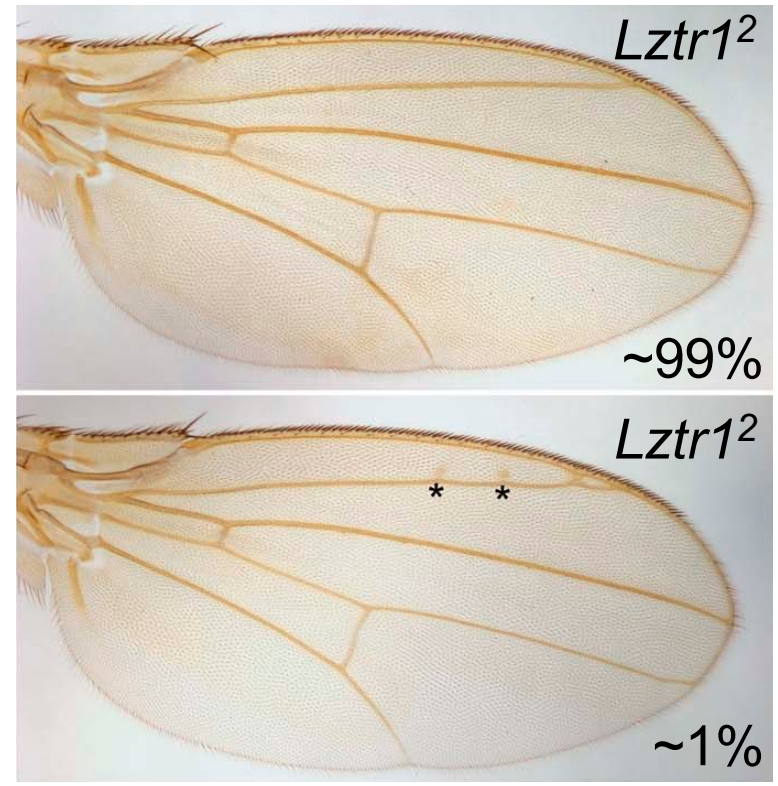

$\mathbf{e}$

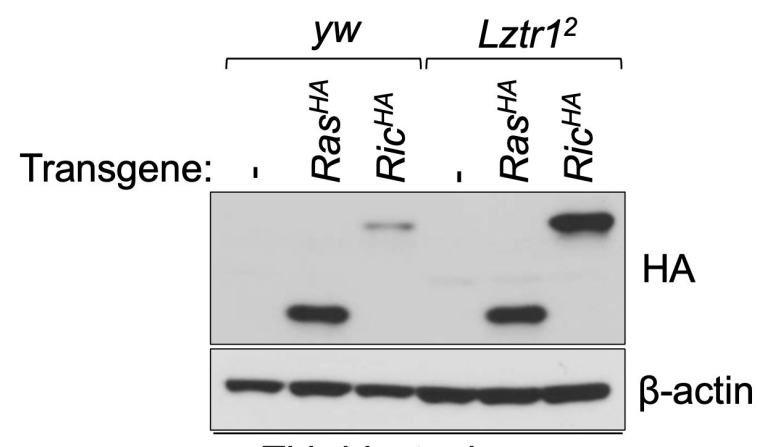

Third-instar larvae 
bioRxiv preprint doi: https://doi.org/10.1101/2022.01.04.474894; this version posted January 5, 2022. The copyright holder for this preprint (which was not certified by peer revjew) is the author/funder, who has granted bioRxiv a license to display the preprint in perpetuity. It is made

a

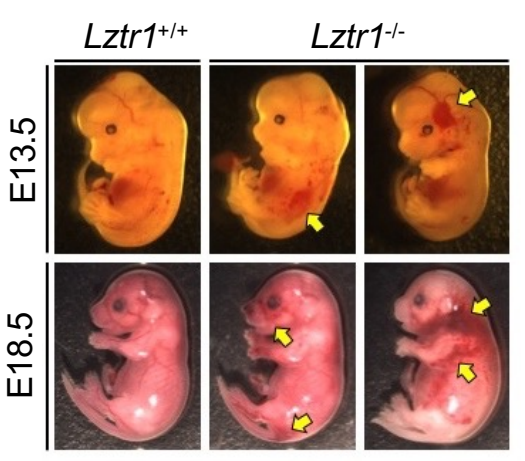

C

Rit1: $+/+\quad+/-$-/-

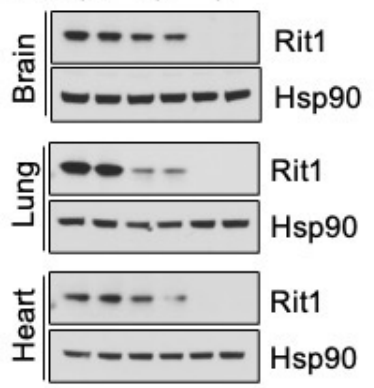

f

Lztri: $+1+-1-+1+-1--1-$

Rit1: $+/++/+-/--/--/-$

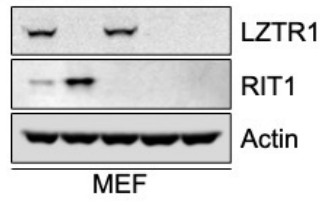

b available under aCC-BY 4.0 International license. Lztr1 Lztr1 $^{+/ /+}$

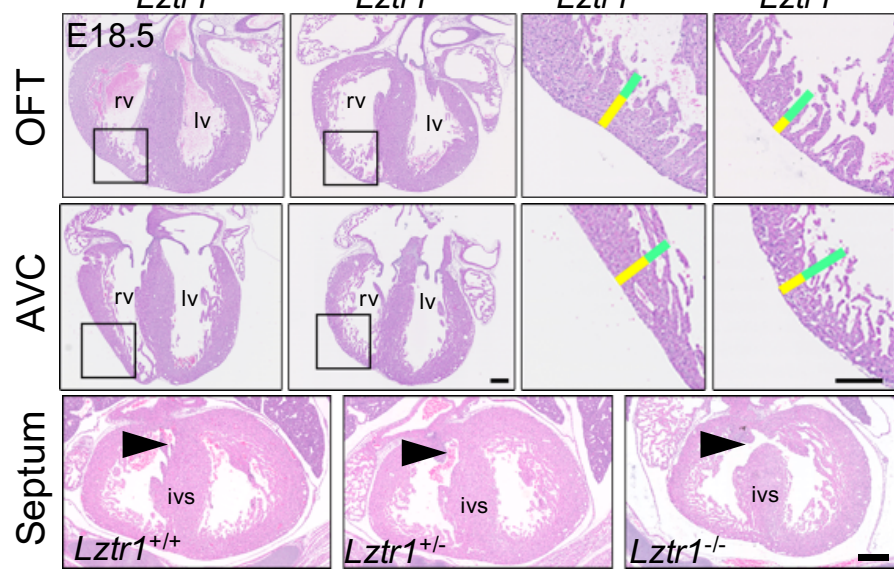

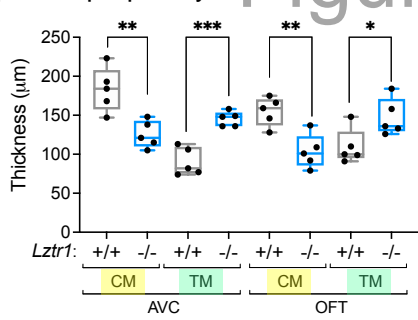

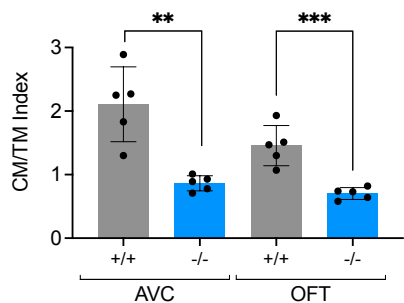

d

$\operatorname{Lztr1}^{+1-} \times \operatorname{Lztr1}^{+1-}$

Rit1 $^{+/+}$

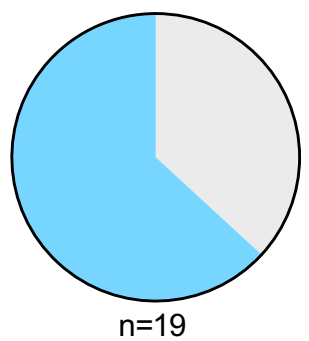

Lztr1+/- $\times$ Lztr1+1-

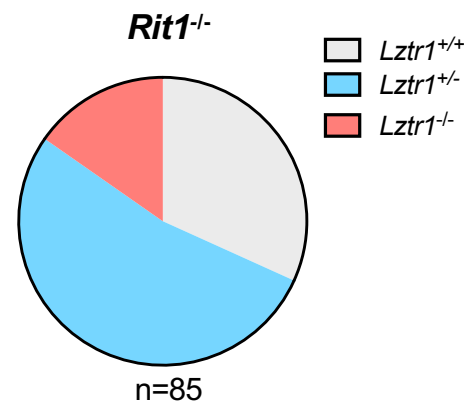

$\mathbf{e}$

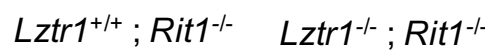

9

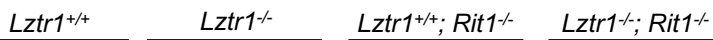
10\% FBS (h): $\overline{015^{\prime} 14824} \overline{015^{\prime} 14824} \frac{L 15^{\prime} 14824}{015^{\prime} 14824}$

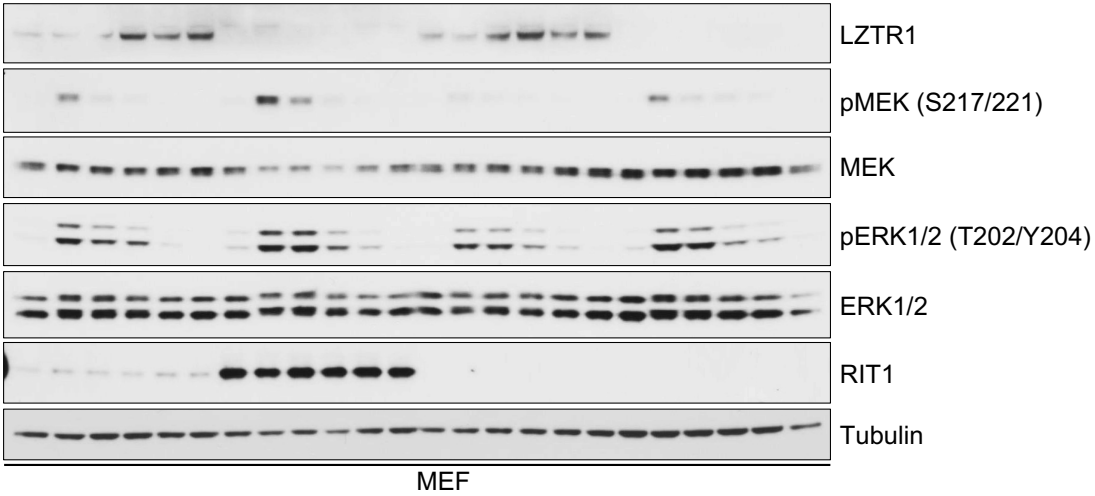




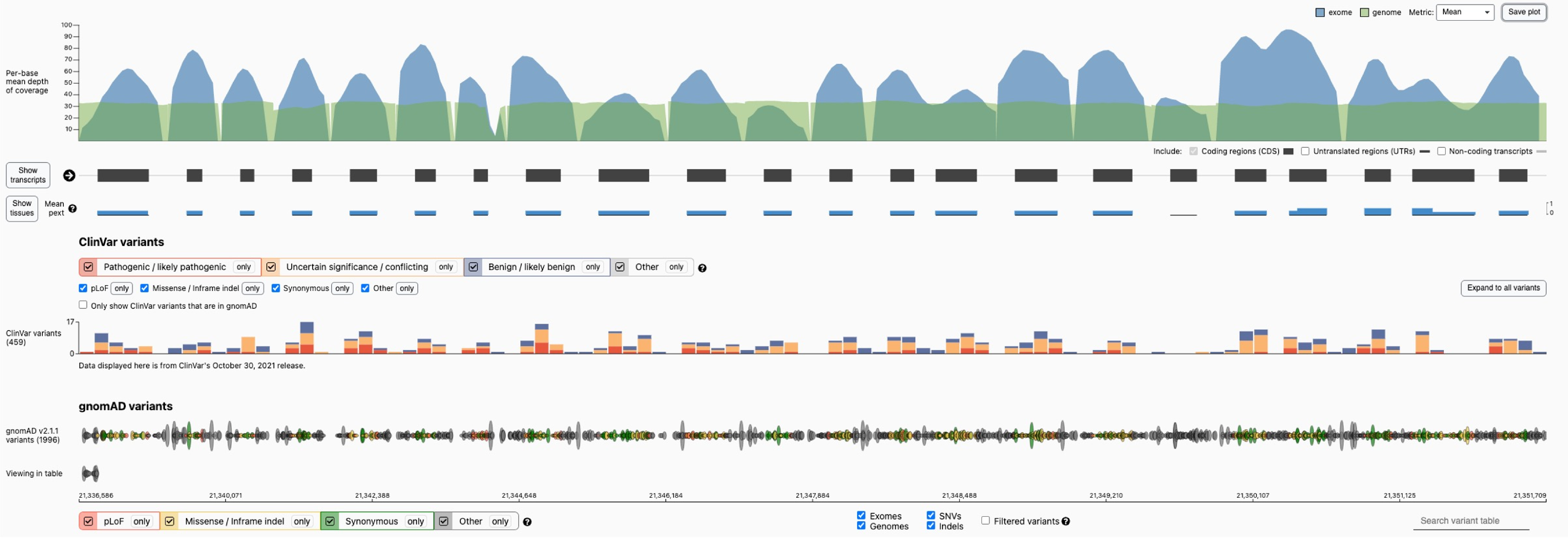

